\title{
Effect of targeted ovarian cancer therapy using amniotic fluid mesenchymal stem cells transfected with enhanced green fluorescent protein-human interleukin-2 in vivo
}

\author{
QI YOU ${ }^{1}$, YUAN YAO $^{1}$, YUANLONG ZHANG $^{2}$, SONGBIN FU $^{3}$, \\ MEI DU ${ }^{4}$ and GUANGMEI ZHANG ${ }^{1}$
}

\begin{abstract}
${ }^{1}$ Department of Gynecology and Obstetrics, The First Affiliated Hospital of Harbin Medical University, Harbin, Heilongjiang 150001; ${ }^{2}$ Department of General Surgery, Harbin Red Cross Central Hospital, Harbin, Heilongjiang 150076; ${ }^{3}$ Department of Genetics, Harbin Medical University, Harbin, Heilongjiang 150081; ${ }^{4}$ Department of Gynecology and Obstetrics, Harbin Maternal and Child Health Care Hospital, Harbin, Heilongjiang 150026, P.R. China
\end{abstract}

Received October 4, 2014; Accepted May 21, 2015

DOI: $10.3892 / \mathrm{mmr} .2015 .4076$

\begin{abstract}
The aim of the present study was to investigate the effect of using amniotic fluid mesenchymal stem cells (AF-MSCs) in targeted ovarian cancer therapy in vivo. AF-MSCs were isolated from human second trimester AF and a plasmid, enhanced green fluorescent protein-human interleukin-2 (pEGFP-hIL-2) was formed. The plasmid was stably transfected into the AF-MSCs and the cells were intravenously injected into ovarian cancer nude mice models. Following stable transfection of the vector, tumor formation, and the expression and activity of hIL-2 were investigated, and microscopic pathological examinations of the tumor were performed. It was found that AF-MSCs exhibited high motility during migration in vivo, and the vector, pEGFP-hIL-2 can be stably transfected into AF-MSCs. Following stable transfection, this type of stem cell is able to successfully transport the therapeutic gene, IL-2, migrate to the ovarian cancer tumor site to secrete the functional IL-2 and treat the tumor. Thus, AF-MSCs may serve as transporters for therapeutic genes targeting ovarian tumor sites and, therefore, be involved in the treatment of tumors.
\end{abstract}

\section{Introduction}

Ovarian cancer is one of the most lethal types of gynecological malignancy. The response to platinum-based chemotherapy is

Correspondence to: Dr Qi You or Dr Guangmei Zhang, Department of Gynecology and Obstetrics, The First Affiliated Hospital of Harbin Medical University, 23 Youzheng Street, Harbin, Heilongjiang 150001, P.R. China

E-mail: yqvicky2001@sina.com

E-mail: drzhangguangmei@163.com

Key words: amniotic fluid, mesenchymal stem cell, ovarian cancer, targeted therapy poor in recurrent patients, thus, the aim of current research is to establish novel therapeutic methods (1).

Cell lines and animal models are valuable research tools for increasing understanding of the molecular mechanisms of neoplasia and for developing potential therapeutic strategies (2). In our previous studies, the SKOV3 human ovarian carcinoma cell line was developed and served as a reliable model of ovarian cancer disease progression (3).

Interleukin (IL)-2 is a cytokine from the cytokine-receptor gamma c-chain family with numerous functions, including stimulating the proliferation of $\mathrm{T}$ cells, inducing the production of natural killer cells, inducing cytotoxic T lymphocyte generation, and facilitating the proliferation and synthesis of immunoglobulins produced by B cells (4). IL-2 is administered to boost immunity in patients with the HIV infection (5) and cancer, including ovarian cancer, metastatic melanoma and renal cell carcinoma (6). IL-2 treatment reliably results in a $16-20 \%$ objective clinical response rate in cancer patients, demonstrating significant durability of responses in selected patients (7).

However, when IL-2 is administered systemically at high doses, the tumor regression is associated with transient side-effects ranging from general malaise, fever, nausea, and vomiting to the more severe reactions, including hepatic dysfunction, increased capillary permeability and decreased systemic vascular resistance $(8,9)$. Therefore, a therapeutic strategy that specifically delivers IL-2 to the tumor location may significantly reduce the required IL-2 dosage, thus alleviating many of the side-effects that are commonly associated with its systemic administration (10).

Human amniotic fluid (AF)-derived stem cells are becoming an important source of cells for cellular therapy. They have the ability to differentiate into cells of all three embryonic germ layers and possess a high proliferation rate (3). During our previous study, mesenchymal stem cells (MSCs) were isolated from human second and third trimester AF and their biological characteristics were demonstrated (3), in addition, a series of experiments were conducted to investigate the possible therapeutic functions of these human AF-MSCs. 
In the present study, IL-2 and the green fluorescent protein (GFP) gene were fused to form a plasmid vector, enhanced GFP-human interleukin-2 (pEGFP-hIL-2), which was stably transfected into AF-MSCs. These stem cells were intravenously injected at various doses into ovarian cancer nude mice models. Tumor formation, and the expression and activity of hIL-2 were analyzed. The pathological examination results were used to identify the therapeutic action of hIL-2 on ovarian cancer. The aim of the current study was to evaluate the migratory potential of AF-MSCs into tumor cells in vivo, as well as determine their function as delivery vehicles for anti-tumor molecules, such as IL-2, to neoplasia sites $(11,12)$.

\section{Materials and methods}

Materials and ethical approval. Unless otherwise stated, all chemicals used in the present study were purchased from Invitrogen Life Technologies (Carlsbad, CA, USA). The current study was conducted in accordance with the Declaration of Helsinki and EU Directive 2010/63/EU. Written and verbal informed consent was obtained from each volunteer, and the study protocol was approved by the Ethics Committee of Harbin Medical University (Harbin, China). All animal experiments were conducted in accordance with the institutional Policies and Guidelines for the Care and Use of Laboratory Animals and all efforts were made to minimize animal suffering.

Isolation and culture of MSCs from human second trimester $A F$. AF samples were harvested from 30 female volunteers who underwent amniotic membrane puncturation. The mean \pm standard deviation of pregnancy duration (fetal age +2 weeks) was $18 \pm 1$ weeks. The AF samples were collected by puncture through the amniotic membrane and MSCs were isolated from the AF of 22 samples.

The samples were centrifuged at $100 \mathrm{x} \mathrm{g}$ for $5 \mathrm{~min}$ and all of the isolated cells were plated in six 35-mm petri dishes containing low-glucose Dulbecco's modified Eagle's medium supplemented with $100 \mathrm{U} / \mathrm{ml}$ penicillin, $0.1 \mathrm{mg} / \mathrm{ml}$ streptomycin, $10 \mathrm{ng} / \mathrm{ml}$ basic fibroblast growth factor, $10 \mathrm{ng} / \mathrm{ml}$ epidermal growth factor (all obtained from PeproTech, Inc., Rocky Hill, NJ, USA) and $20 \%$ fetal bovine serum. The medium was renewed after the cells had been incubated at $37^{\circ} \mathrm{C}$ in a humidified atmosphere of $5 \%$ carbon dioxide for 7 days; any non-adhering cells were removed. After expansion to $70 \%$ confluence, the cells were harvested by trypsinization and subcultured. Markers of AF-MSCs were analyzed by reverse-transcription polymerase chain reaction (RT-PCR), flow cytometry and differentiation potential assays (4).

EGFP gene transfection into AF-MSCs. For transfection of the EGFP gene into the AF-MSCs, $40 \mu 1$ Lipofectamine $^{\mathrm{TM}} 2000$ and $3 \mu \mathrm{g}$ pEGFP-N1 (Clontech Laboratories, Inc., Mountain View, CA, USA) were dissolved into $2 \mathrm{ml}$ antibiotic-free AF-MSC growth medium. The AF-MSCs $\left(5 \times 10^{4}\right.$ cells $)$ were passaged four times and cultured in the1-ml Lipofectamine $^{\mathrm{TM}}$ 2000/pEGFP-N1 mixture for $20 \mathrm{~h}$. The mixture was removed and the cells were cultured for 1 week with an AF-MSC culture medium containing G418 $(350 \mu \mathrm{g} / \mathrm{ml})$. The expression of EGFP in the cells was monitored under an

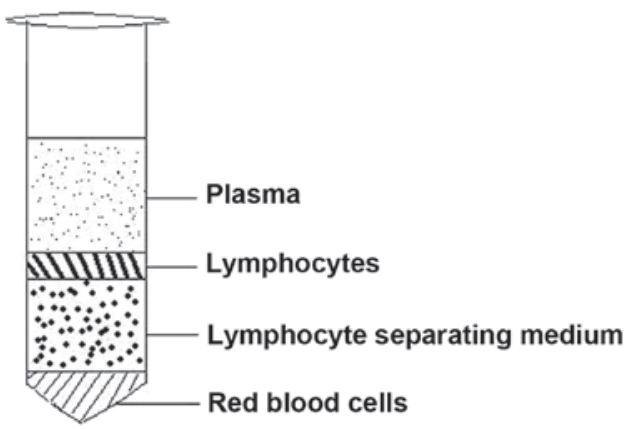

Figure 1. Blood was separated into four layers using lymphocyte separating medium.

inverted UV microscope (TE2000-U; Nikon, Inc., Tokyo, Japan). One week later, the stable transfectants were selected and the transfected AF-MSCs were passaged as a single-cell suspension. RNA was extracted from the fifth passage of the EGFP gene-transfected AF-MSCs, and used for RT-PCR analysis of the cell markers.

Absence of tumor formation. The EGFP-transfected human AF-MSCs were injected into the rear leg muscles of 4-week-old male SCID beige mice (CB17.B6-Prkd ${ }^{\text {cscid }}$ Lyst $^{\text {bg }} / \mathrm{CrlVr}$; Heze Better Biotechnology co., Ltd., Shandong, China). Fifteen mice were injected with $3 \times 10^{6}$ cells per animal. Three months after the injection, the injected muscles were subjected to histological examination and no tumor was observed.

$R T$-PCR. Total RNA was extracted from the transfected AF-MSCs using Tri Reagent according to the manufacturer's instructions, and RT-PCR was performed using a One Step RT-PCR kit with selective DNA primers for the genes, octamer-binding protein 4 (OCT4) and $\beta$-actin as follows: Sense, 5'-CGTGAAGCTGGAGAAGGAGAAGCTG-3' and antisense, 5'-CAAGGGCCGCAGCTTACACATGTTC-3' for OCT4 (length, 247 bp); and sense, 5'-TGGCACCACACCTTCTACAATGAGC-3' and antisense, 5'-GCACAGCTTCTCCTTAATGTCACGC-3' for $\beta$-actin (length, 396 bp). NTERA-2 cl. D1 cells from a pluripotent human testicular embryonic carcinoma cell line (ATCC CRL-1973) and MRC-5 cells derived from human diploid fibroblasts from fetal lung tissue (ATCC CCL-171) served as positive and negative controls, respectively, for OCT4 expression analysis.

hIL-2 gene extraction. Peripheral blood samples $(4 \mathrm{ml})$ were obtained from the volunteers and mixed with Hanks' balanced salt solution (HBSS) at a 1:1 volume. The mixture was added to $8 \mathrm{ml}$ human lymphocyte separating medium and the samples were centrifuged at $150 \mathrm{x}$ g for $15 \mathrm{~min}$. The cells were separated into four layers as follows: First layer, plasma; second layer, lymphocytes and monocytes; third layer, lymphocyte separating medium; and fourth layer, red blood cells (Fig. 1).

The lymphocytes from layer two were washed with HBSS, and broken down using TRIzol. The mixture was kept still for 5 min, then mixed with chloroform (volume, 1:0.2) and centrifuged at $225 \mathrm{xg}$ for $15 \mathrm{~min}$ at $4^{\circ} \mathrm{C}$. Isopropyl alcohol was added to the mixture at a volume of $1: 1.2$, maintained at $4^{\circ} \mathrm{C}$ for $1 \mathrm{~h}$ 

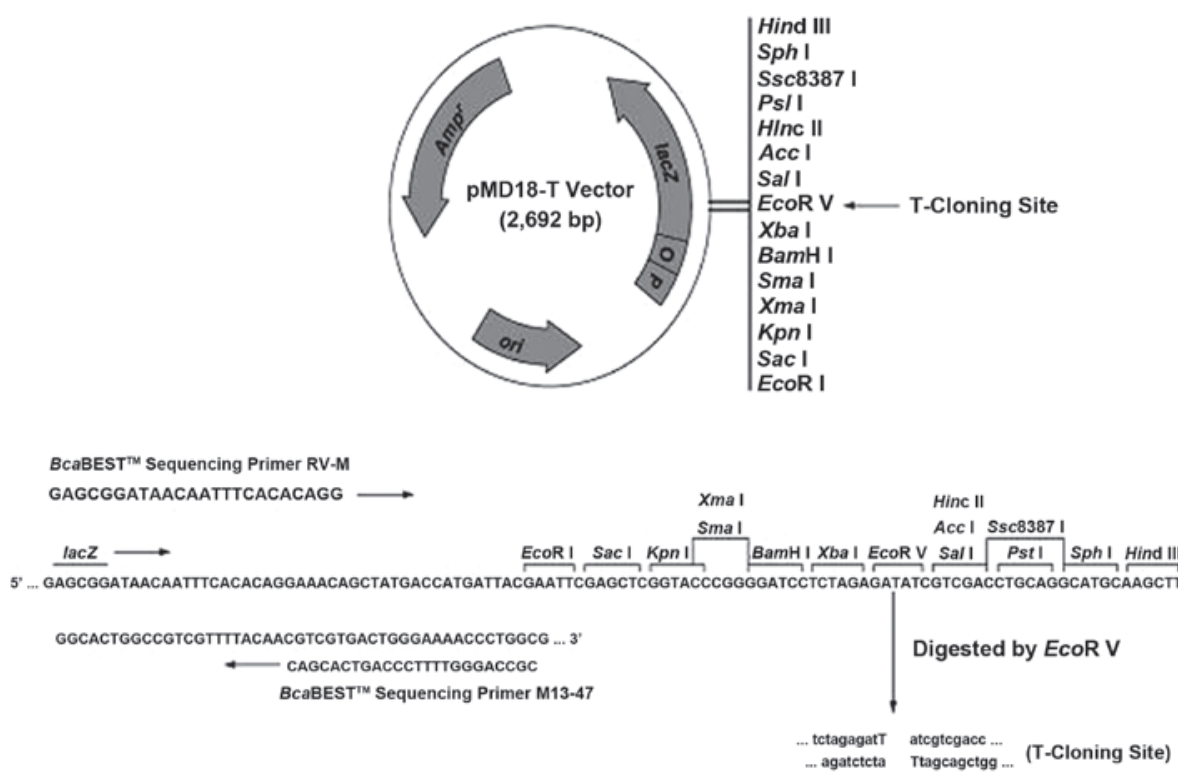

Figure 2. Scheme depicting the plasmid pMD18-T vector which is linked with the interleukin-2 gene in the present study.

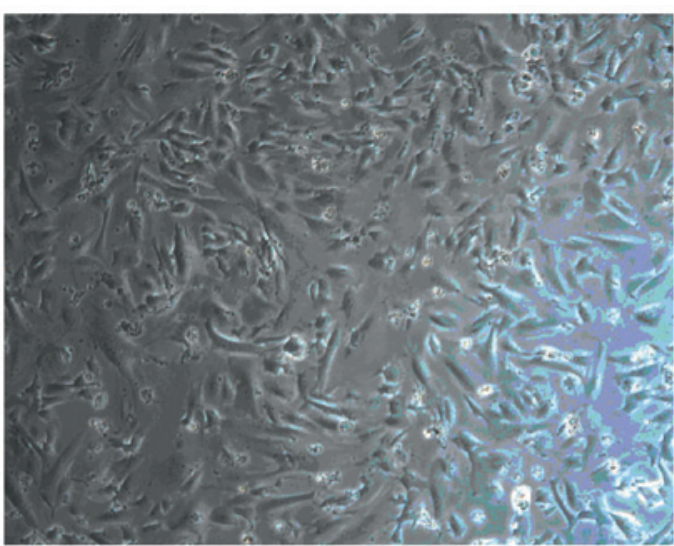

Figure 3. Human AF mesenchymal stem cells from second trimester AF (magnification, x50). AF, amniotic fluid.

and centrifuged at $225 \mathrm{xg}$ for $10 \mathrm{~min}$, then washed with alcohol and mixed with $20 \mu \mathrm{l}$ diethylpyrocarbonate. The mixture was then stored at $-80^{\circ} \mathrm{C}$ in a refrigerator. RT-PCR was performed to synthesize cDNA with the above-mentioned sample.

The DNA primers were designed according to Genbank (http://www.ncbi.nlm.nih.gov/genbank/). The primers were as follows: Upstream, 5'GGAATTCATGTACAGGATG3' for passage 1 (P1); and downstream, 5'GACTGAACTCAGCTGG3' for P2 (synthesized by Invitrogen Life Technologies). Following the PCR reaction and agarose gel electrophoresis, a DNA product (length, 462 bp) was obtained and recycled.

hIL-2 gene segment cloning and identification. The purified product was combined with a pMD18-T Simple Vector (Fig. 2) and $10 \mu \mathrm{l}$ of the above-mentioned DNA product was mixed with $100 \mu 1$ JM109 competent cells (provided by Dr Yin Zhe, Department of Microbiology, China Agricultural University, Harbin, China) in an ice bath. The mixture was placed in ice for $30 \mathrm{~min}$, heated at $42^{\circ} \mathrm{C}$ for $90 \mathrm{sec}$ and placed in an ice bath for a further 1-2 min. Following this, $800 \mu 1$ medium without antibiotics was added into the above-mentioned sample and centrifuged at $22 \times \mathrm{g}$ for $40 \mathrm{~min}$ at $37^{\circ} \mathrm{C}$. After centrifugation, $\sim 100 \mu \mathrm{l}$ of the supernatant was kept and spread on Luria-Bertani (LB) agar plates with Penbritin, X-gal and osopropyl $\beta$-D-1-thiogalactopyranoside (100 $\mu \mathrm{g} / \mathrm{ml}$ of each). Twenty-four hours later, the LB mixture inoculated with Penbritin was agitated at $37^{\circ} \mathrm{C}$ for $8 \mathrm{~h}$. The plasmid was extracted according to the manufacturer's instructions. Following PCR amplification, the sample was digested with EcoRI and SalI, and agarose gel electrophoresis was performed. The length of the DNA fragment obtained was $462 \mathrm{bp}$, which was verified by Invitrogen Life Technologies, and the recombinant plasmid was designated pMD-hIL-2.

Transient transfection of pMD-hIL-2 into AF-MSCs. The AF-MSCs were inoculated during the logarithmic growth phase and plated in 6-well petri dishes. When the cells reached $80-90 \%$ confluence, the cells were washed with Opti-minimal essential medium cell culture fluid (Invitrogen Life Technologies), and the recombinant plasmid, pMD-hIL-2 was transfected into the AF-MSCs using Lipofectamine ${ }^{\mathrm{TM}} 2000$ reagent, according to the manufacturer's instructions. After $48 \mathrm{~h}$, the cells were selected using $500 \mu \mathrm{g} / \mathrm{ml} \mathrm{G} 418$; selection continued for 14 days, and the monoclonal cells were isolated and cultured. hIL-2 gene expression activity was detected in the cells using an hIL-2 detection kit. GFP gene expression was detected under a fluorescence microscope (Eclipse 600; Nikon, Inc.).

Establishing an ovarian cancer animal model. When the SKOV3 ovarian cancer cells (cultured in vitro) reached $70 \%$ confluence, the cells were washed with PBS, digested with trypsin and washed again with PBS. The cells were then suspended in PBS and the concentration of the cells was adjusted to $2 \times 10^{7}$ cells $/ \mathrm{ml}$. Each specific pathogen free (SPF) grade nu/nu-BALB/c nude mouse ( $n=50$; age, 4 weeks) was administered with $0.2 \mathrm{ml}\left(4 \times 10^{6}\right.$ cells) of the suspended cells, 


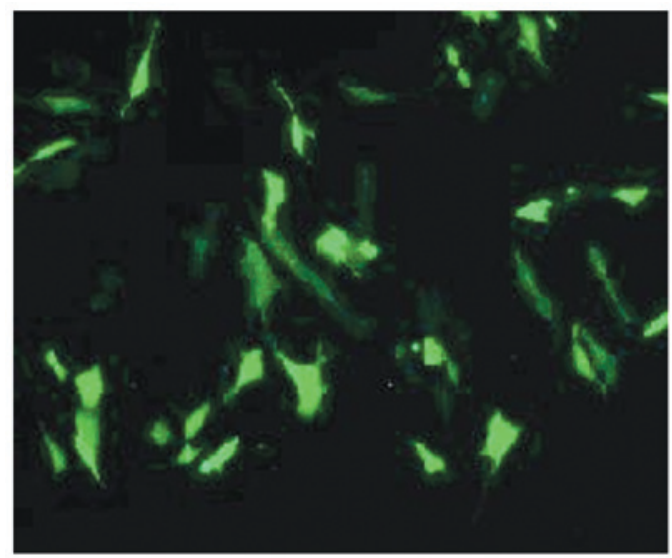

Figure 4. Enhanced green fluorescent protein-transfected amniotic fluid mesenchymal stem cells (magnification, $\mathrm{x} 50$ ).

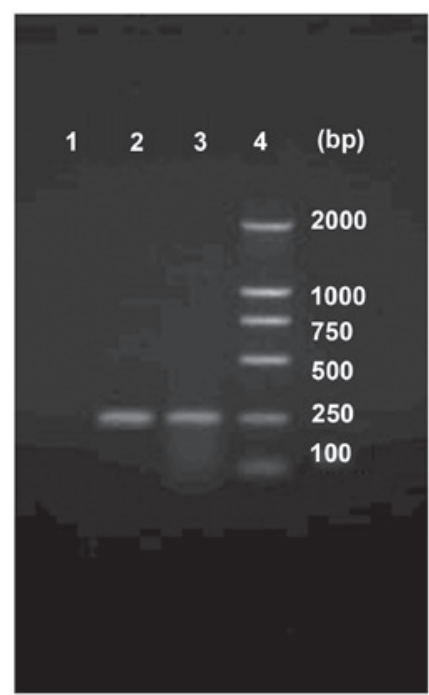

Figure 5. Enhanced green fluorescent protein-transfected amniotic fluid mesenchymal stem cells express OCT4. Lane 1, negative control; lane 2, positive control; lane 3, OCT4 mRNA; lane 4, marker. OCT4, octamer-binding protein 4.

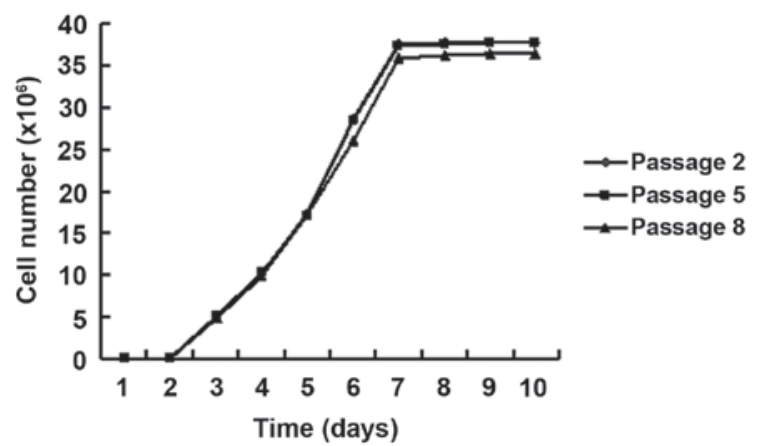

Figure 6. Growth curve of enhanced green fluorescent protein-transfected human amniotic fluid mesenchymal stem cell colonies at passage two, five and eight.

which were subcutaneously injected into the scapula region. This was performed in particularly clean conditions to ensure the procedure was aseptic.

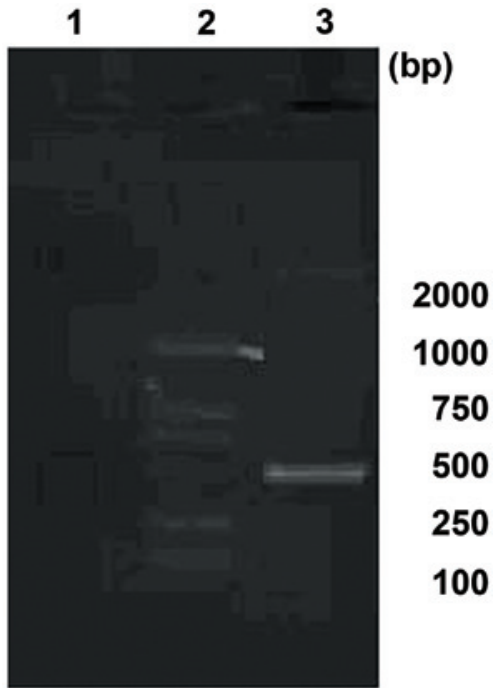

Figure 7. Reverse transcription polymerase chain reaction analysis of hIL-2 gene expressed. Lanes: 1, water negative control; 2, marker; 3, hIL-2. hIL-2, human interleukin-2.

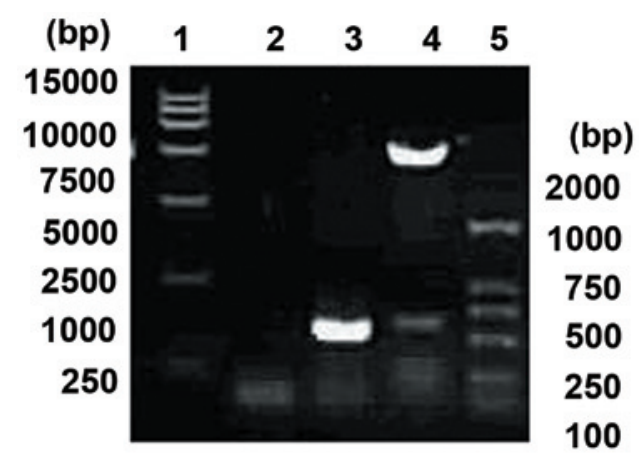

Figure 8. Identification of the recombinant plasmid, pEGFP-hIL-2. Lane 1, 15,000-bp marker; lane 2, water negative control; lane 3, hIL-2; lane 4, pEGFP-hIL-2; lane 5, 2,000-bp marker. pEGFP-hIL-2, enhanced green fluorescent protein-human interleukin-2.

Targeted ovarian cancer therapy using AF-MSCs. When the subcutaneously transplanted tumor reached $1 \mathrm{~cm}$ in diameter, $0.2 \mathrm{ml}$ of AF-MSCs transiently transfected with the recombinant plasmid, pMD-hIL-2 were injected through the caudal vein of each ovarian cancer mouse at $2 \times 10^{7}$ cells $/ \mathrm{ml}$. Two control groups were established; a sodium chloride group and a pEGFP-hIL-2 group, with 15 mice in each group. The littermates were raised in an SPF animal center under identical conditions. The body weight and tumor size of each mouse was noted daily and every seven days, one mouse from each group was sacrificed with ether. The fluorescence distribution was analyzed and pathological sections of the tumor were obtained, which were used to detect ovarian cancer cell apoptosis under an electron microscope (JEM-1200EX; JEOL, Ltd., Tokyo, Japan).

\section{Results}

Characteristics of EGFP-transfected AF-MSCs. Human AF-MSCs were successfully isolated from second trimester AF (Fig. 3) and the EGFP gene was transfected into the 

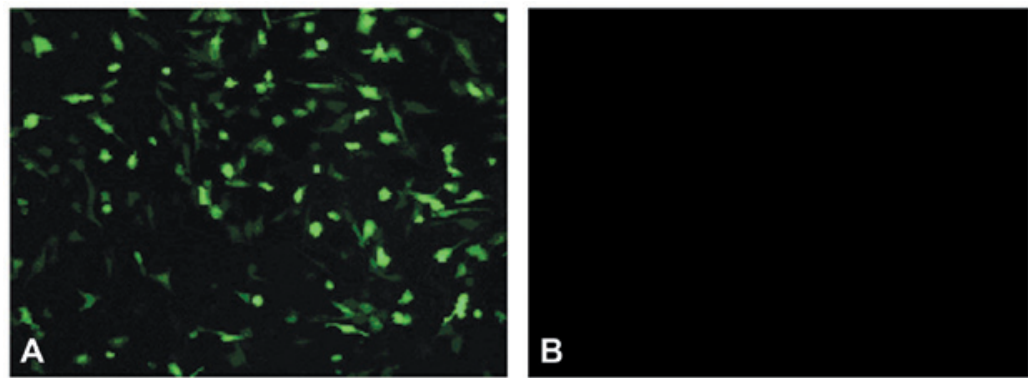

Figure 9. (A) Stable transfection of the pEGFP-hIL-2 vector into AF-MSCs (magnification, x50). (B) Untransfected AF-MSCs (magnification, x50). pEGFP-hIL-2, enhanced green fluorescent protein-human interleukin-2; AF-MSCs, amniotic fluid mesenchymal stem cells.

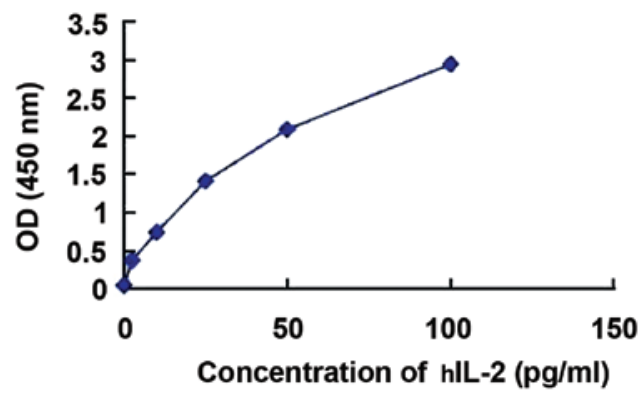

Figure 10. Expression of the hIL-2 gene following transfection into amniotic fluid mesenchymal stem cells. OD, optical density; hIL-2, human interleukin-2.
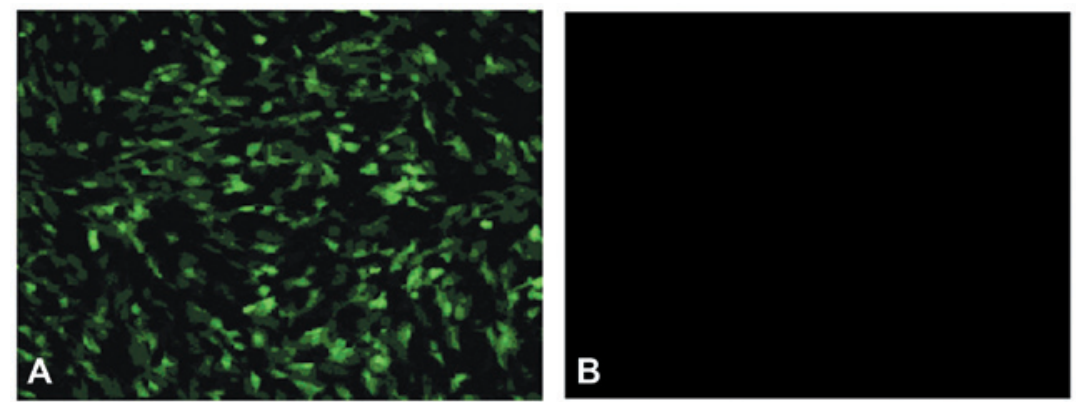

Figure 11. (A) Stable transfection of pEGFP-hIL-2 vector into AF-MSCs (magnification, x50). (B) Untransfected AF-MSCs (magnification, x50). pEGFP-hIL-2, enhanced green fluorescent protein-human interleukin-2; AF-MSCs, amniotic fluid mesenchymal stem cells.

AF-MSCs via lipofection (Fig. 4; EGFP gene transfection efficiency, 30\%). The EGFP-transfected AF-MSCs were found to express OCT4 (Fig. 5). Furthermore, EGFP was expressed in all of the transgenic cells (following selection of the stably transfected cell lines) and could be readily visualized; this expression was maintained over ten passages. The growth curve of the EGFP-transfected AF-MSCs exhibited almost the same characteristics as untransfected AF-MSCs. From day three, the cells entered the logarithmic growth stage and the growth peak was observed on day seven. The doubling time was $30.5 \mathrm{~h}$. No significant difference was identified between the different passages (Fig. 6).

Ovarian cancer nude mice model formation. SKOV3 cells were subcutaneously injected into the right scapula region of each nude mouse. After one week, no redness, swelling or ulcerations were observed in the injection region and the diameter of the subcutaneously transplanted tumor was $0.1-0.3 \mathrm{~cm}$. The tumor grew at a rate of $1 \mathrm{~cm}$ per month.
hIL-2 gene extraction and identification. Human lymphocytes were separated from the peripheral blood of healthy adults, the total RNA was extracted and following RT-PCR, electrophoresis and DNA purification, the hIL-2 gene was obtained (Fig. 7). After combining with a pMD18-T Simple Vector, a sequencing test indicated that the sequence was correct.

Identification of the hIL-2 gene recombinant plasmid, PEGFP-hIL-2. The recombinant plasmid, pEGFP-hIL-2 was digested by the two enzymes, EcoRI and SalI, and following agarose gel electrophoresis an exogenous fragment (length, 462 bp) was identified (Fig. 8), the result was indicated by Invitrogen Life Technologies.

Transient transfection of the recombinant plasmid into AF-MSCs. The recombinant plasmid-transfected AF-MSCs are shown in Fig. 9A by green fluorescence. Following continuous culture for two weeks, the green fluorescence was stable; however, the untransfected AF-MSCs didn't fluoresce (Fig. 9B). 

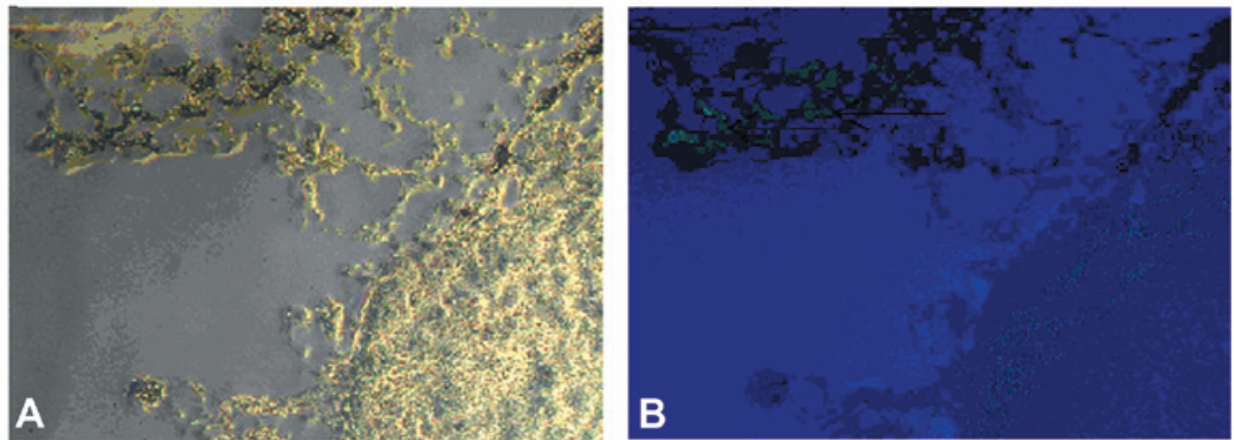

Figure 12. GFP-labeled amniotic fluid mesenchymal stem cells surrounding the tumor mass. The GFP-specific signal as observed by (A) phase contrast microscopy (magnification, x50) and (B) fluorescence microscopy (magnification, x50). GFP, green fluorescent protein.

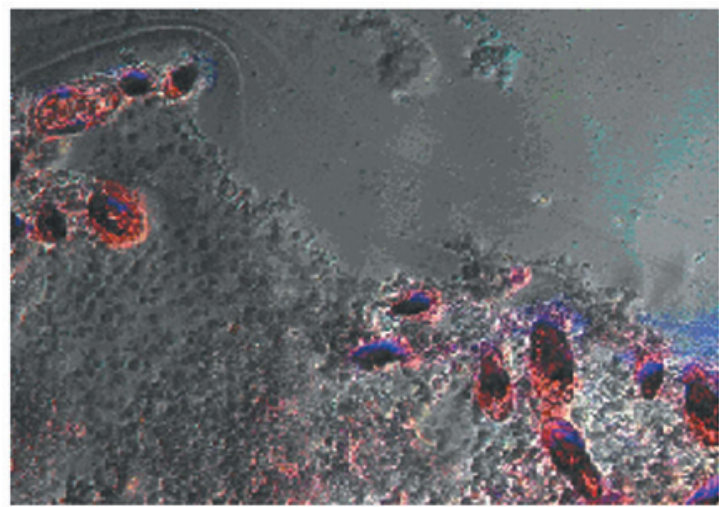

Figure 13. Hematoxylin and eosin staining of the tumor mass and the surrounding amniotic fluid mesenchymal stem cells (magnification, x200).
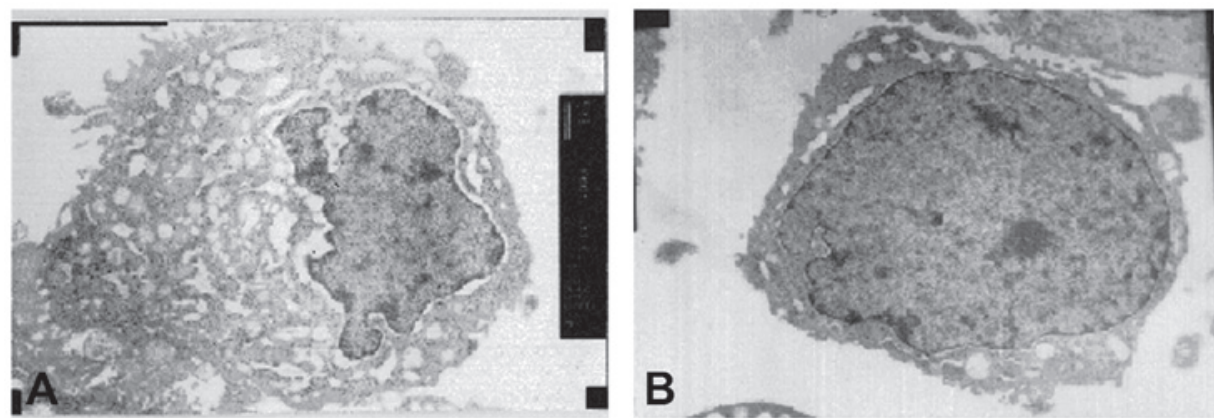

Figure 14. Transmission electron microscopic observation of apoptosis in SKOV3 cells (magnification, $\mathrm{x} 4,000$ ). (A) Apoptotic SKOV3 cell displaying a swollen endoplasmic reticulum and (B) SKOV3 cell from the control group (magnification, $\mathrm{x} 4,000$ ).

hIL-2 gene expression detection. After the recombinant plasmid-transfected AF-MSCs became stable, a hIL-2 testing kit was used to examine the clear supernatant of these cells; the clear supernatant of the untransfected AF-MSCs served as the negative control.

A standard substance dilution was used and the optical density (OD) value was measured at $450 \mathrm{~nm}$. The standard curve is shown in Fig. 10. The OD value of the clear supernatant of the recombinant plasmid-transfected AF-MSCs at $450 \mathrm{~nm}$ was 1.103 , while that of the untransfected cells was 0.052 . The OD value of the tested sample was more than five times higher than that of the negative control, therefore the result was positive according to the kit instructions. The following formula was used: $\mathrm{y}=\mathrm{ax} \mathrm{x}^{2}+\mathrm{bx}+\mathrm{c}$ as well as the standard curve, and the hIL-2 concentration in the treated sample was found to be $17.258 \mathrm{pg} / \mathrm{ml}$.

GFP gene expression detection. The recombinant plasmid-transfected AF-MSCs emitted stable green fluorescence under a fluorescence microscope, which demonstrated the expression of the GFP gene (Fig. 11A), while the untransfected cells did not fluoresce (Fig. 11B).

Pathological examination of ovarian cancer tissue and surrounding tissue. Six weeks after the recombinant plasmid-transfected AF-MSCs were injected into the ovarian cancer mouse model, green fluorescence was apparent around the tumor tissue, whereas the other issue emitted only slight 
fluorescence. No other tumors formed in the animal body. After the animals were sacrificed, the tumor tissue and the surrounding tissue were immediately excised and frozen sections were made. Green fluorescence was observed from the AF-MSCs around the tumor tissue, as shown in Fig. 12. Acetone was used to fix the sections and they were stained with hematoxylin and eosin (H\&E). Fusiform shaped AF-MSCs were demonstrated by the $\mathrm{H} \& \mathrm{E}$, as well as anachromasis (Fig. 13).

Ultrastructure examination of ovarian cancer cells. The ovarian cancer tissue of the treatment group (nude mice that were injected with the pEGFP-hIL-2 recombined plasmid) was fixed using glutaraldehyde, and the ultrastructure of SKOV3 ovarian cancer cells was observed under a transmission electron microscope. The reductus of the nuclear membrane was observed to be depressed, chromatin was condensed, more compact, concentrated and parted, and there were more layers of endoplasmic reticulum surrounding the nucleus. Furthermore, the endoplasmic reticulum had become swollen and expanded, as shown in Fig. 14A. The ultrastructure of the ovarian cancer cells from the untreated group (observed by transmission electron microscope) are presented in Fig. 14B.

\section{Discussion}

Ovarian cancer is a common type of malignant tumor, and the rate of case fatalities is the highest among the gynecological malignant tumors. Ovarian cancer threatens the physical and mental health of females, as well as their quality of life. As the majority of patients have reached an advanced stage when they are diagnosed with ovarian cancer, abdominal implantation and metastasis occurs, resulting in a rapid decrease of the quality of life. The five-year survival rate is $\sim 30 \%$. Over the past 30 years, gynecological oncological scientists worldwide have attempted chemotherapy, radiotherapy and gene therapy on the basis of cytoreductive surgery, and this has been the predominant therapeutic method for ovarian cancer. However, relapse and metastasis continue to be observed following surgery in most advanced-stage ovarian cancer patients (13).

IL-2 administration stimulates existent forkhead box P3 and T cell proliferation, and may promote regulatory $\mathrm{T}$ cell tumor trafficking in patients with ovarian cancer (14). Certain physicians have attempted to use IL-2 to cure patients suffering with metastasized ovarian cancer; however, high concentrations of IL-2 are required to obtain a therapeutic response. Although the treatment has a curative effect, there are too many side-effects; therefore, targeted gene therapy may act as a substitute for the traditional treatment methods. Verification of the principle and efficiency of this approach, to act locally at the tumor microenvironment and inhibit malignant cell growth in vivo, was provided in other types of cancer using MSCs derived from adult sources, such as bone marrow (BM) (15). Specifically, homing of MSCs following systemic or local administration has been investigated in a variety of tumor animal models, including models of lung metastasis (16), melanoma (17), and brain glioma (18), amongst others. Collectively, these studies supported the hypothesis that exogenously administrated BM-MSCs preferentially engrafted at the tumor site by contributing to the population of the stromal fibroblasts, thus forming the tumor's microenvironment (19). This important characteristic determined stem cells to be of importance within targeted tumor therapy, particularly in patients that have relapsed or are exhibiting a metastatic tumor, as targeting the tumor tissue avoids undesirable side-effects in other tissues, including liver, kidney, spleen and lung. AF-MSCs may be considered as a powerful tool for gene therapy and tissue repair in the clinical setting, due to their high proliferative activity and chromosomal stability. AF-MSCs exhibited chromosomal stability with no karyotypic abnormalities, even following high numbers of passages, and possessed long telomeres and exhibited no tumorigenic effect in vivo (20). In the present study, AF-MSCs transduced with GFP were analyzed in a mouse ovarian cancer model to evaluate the migratory properties in vivo. GFP was expressed in all of the transgenic cells (following selection of the stably transfected cell lines), and notably, this expression was maintained over numerous passages. The localization of AF-MSCs at the tumor site indicated that these cells, similarly to BM-MSCs (21), are able to reach the extravascular space and contribute to the development of tumor connective stroma. It was found in the present study that intravenously injected AF-MSCs, that stably express hIL-2, are able to trace the subcutaneously transplanted ovarian tumor cells, and secrete IL-2 locally, resulting in the apoptosis of the tumor cells. The current study provides an important method for targeted gene therapy to treat ovarian cancer. However, the migratory properties of AF-MSCs in an ovarian cancer mouse model in vivo requires further evaluation for the potential signaling mechanisms that may be involved.

\section{Acknowledgements}

The present study was supported by the post-doctoral financial assistance of the Heilongjiang province (Heilongjiang, China; grant no. LBH-Z11082), the post-doctoral financial assistance of China (grant no. 2013M531073), the Opening Project of the Key Laboratory of Medical Genetics (Harbin Medical University, Heilongjiang Higher Education Institutions, Harbin 150081, China) and the Special Prophase Project (the 973 Project; no. 2012 CB526700).

\section{References}

1. Smolle E, Taucher V, Pichler M, Petru E, Lax S and Haybaeck J: Targeting signaling pathways in epithelial ovarian cancer. Int $\mathbf{J}$ Mol Sci 14: 9536-9555, 2013.

2. Crallan RA, Georgopoulos NT and Southgate J: Experimental models of human bladder carcinogenesis. Carcinogenesis 27: 374-381, 2006.

3. You Q, Cai L, Zheng J, Tong X, Zhang D and Zhang Y: Isolation of human mesenchymal stem cells from third-trimester amniotic fluid. Int J Gynaecol Obstet 103: 149-152, 2008.

4. Waldmann TA: The biology of interleukin-2 and interleukin-15: Implications for cancer therapy and vaccine design. Nat Rev Immunol 6: 595-601, 2006.

5. Sereti I, Anthony KB, Martinez-Wilson H, Lempicki R, Adelsberger J, Metcalf JA, Hallahan CW, Follmann D, Davey RT, Kovacs JA, et al: IL-2-induced CD4+ T-cell expansion in HIV-infected patients is associated with long-term decreases in T-cell proliferation. Blood 104: 775-780, 2004.

6. Tourani JM, Pfister C, Tubiana N, Ouldkaci M, Prevot G, Lucas V, Oudard S, Malet M, Cottu P, Ferrero JM, et al; Subcutaneous Administration Propeukin Program Cooperative Group: Subcutaneous interleukin-2 and interferon alfa administration in patients with metastatic renal cell carcinoma: Final results of SCAPP III, a large, multicenter, phase II, nonrandomized study with sequential analysis design - the Subcutaneous Administration Propeukin Program Cooperative Group. J Clin Oncol 21: 3987-3994, 2003. 
7. Yang T, Wall EM, Milne K, Theiss P, Watson P and Nelson BH: $\mathrm{CD} 8+\mathrm{T}$ cells induce complete regression of advanced ovarian cancers by an interleukin (IL)-2/IL-15 dependent mechanism. Clin Cancer Res 13: 7172-7180, 2007.

8. Vlad AM, Budiu RA, Lenzner DE, Wang Y, Thaller JA, Colonello K, Crowley-Nowick PA, Kelley JL, Price FV and Edwards RP: A phase II trial of intraperitoneal interleukin-2 in patients with platinum-resistant or platinum-refractory ovarian cancer. Cancer Immunol Immunother 59: 293-301, 2010.

9. Lee JM, Yoon SH, Kim HS, Kim SY, Sohn HJ, Oh ST, Oh IH and Kim TG: Direct and indirect antitumor effects by human peripheral blood lymphocytes expressing both chimeric immune receptor and interleukin-2 in ovarian cancer xenograft model. Cancer Gene Ther 17: 742-750, 2010.

10. Kang TH, Mao CP, He L, Tsai YC, Liu K, La V, Wu TC and Hung CF: Tumor-targeted delivery of IL-2 by NKG2D leads to accumulation of antigen-specific CD8+ T cells in the tumor loci and enhanced anti-tumor effects. PLoS One 7: e35141, 2012.

11. Le Page C, Génin P, Baines MG and Hiscott J: Interferon activation and innate immunity. Rev Immunogenet 2: 374-386, 2000.

12. Gao Y, Yao A, Zhang W, Lu S, Yu Y, Deng L, Yin A, Xia Y, Sun B and Wang X: Human mesenchymal stem cells overexpressing pigment epithelium-derived factor inhibit hepatocellular carcinoma in nude mice. Oncogene 29: 2784-2794, 2010.

13. Lynch HT, Casey MJ, Snyder CL, Bewtra C, Lynch JF, Butts M and Godwin AK: Hereditary ovarian cancer: Heterogeneity, molecular genetics, pathology, and management. Mol Oncol 3: 97-137, 2009.
14. Wei S, Kryczek I,Edwards RP, Zou L, Szeliga W, Banerjee M, Cost M, Cheng P, Chang A, Redman B, et al: Interleukin-2 administration alters the CD4+FOXP3+ T-cell pool and tumor trafficking in patients with ovarian carcinoma. Cancer Res 67: 7487-7494, 2007.

15. Yong RL, Shinojima N, Fueyo J, Gumin J, Vecil GG, Marini FC, Bogler O, Andreeff $\mathrm{M}$ and Lang FF: Human bone marrow-derived mesenchymal stem cells for intravascular delivery of oncolytic adenovirus Delta24-RGD to human gliomas. Cancer Res 69: 8932-8940, 2009.

16. Studeny M, Marini FC, Champlin RE, Zompetta C, Fidler IJ and Andreeff M: Bone marrow-derived mesenchymal stem cells as vehicles for interferon-beta delivery into tumors. Cancer Res 62: 3603-3608, 2002.

17. Ren C, Kumar S, Chanda D, Chen J, Mountz JD and Ponnazhagan S: Therapeutic potential of mesenchymal stem cells producing interferon-alpha in a mouse melanoma lung metastasis model. Stem Cells 26: 2332-2338, 2008.

18. Bexell D, Scheding S and Bengzon J: Toward brain tumor gene therapy using multipotent mesenchymal stromal cell vectors. Mol Ther 18: 1067-1075, 2010.

19. Spaeth E, Klopp A, Dembinski J, Andreeff M and Marini F: Inflammation and tumor microenvironments: Defining the migratory itinerary of mesenchymal stem cells. Gene Ther 15: 730-738, 2008

20. De Coppi P, Bartsch G Jr, Siddiqui MM, Xu T, Santos CC, Perin L, Mostoslavsky G, Serre AC, Snyder EY, Yoo JJ, et al: Isolation of amniotic stem cell lines with potential for therapy. Nat Biotechnol 25: 100-106, 2007.

21. Ren C, Kumar S, Chanda D, Chen J, Mountz JD and Ponnazhagan S: Therapeutic potential of mesenchymal stem cells producing interferon-alpha in a mouse melanoma lung metastasis model. Stem Cells 26: 2332-2338, 2008. 